\title{
AC 2010-2204: DATA IN DEPTH: WEB 3-D TECHNOLOGIES PROVIDE NEW APPROACHES TO THE PRESENTATION OF COURSE CONTENT
}

\section{Charles Lesko, East Carolina University}

Charles Lesko is an Assistant Professor in the Department of Technology Systems, College of Technology \& Computer Science at East Carolina University. He received his BS at the US Naval Academy; he holds a MS in Forensics from National University and a second MS in Computer Information Systems from Boston University; his $\mathrm{PhD}$ is in Applied Management from Walden University. His current teaching and research regime focus on strategic technology management and communication, information technology project management, and virtual reality technology use in the workplace. His career focus has been on managing and leading technological innovation in the workplace. He has over (15) years of experience in networking, systems integration and the project management fields. His prior experience base also includes (6) years as a full-time faculty instructor and (12) year's service as an officer in the United States Marine Corps. Address: College of Technology \& Computer Science, 212 Science and Technology Bldg, East Carolina University, Greenville, NC 27858; email: leskoc@ecu.edu; phone: 252-737-1907.

\section{John Pickard, East Carolina University}

John Pickard is an Instructor in the Department of Technology Systems, College of Technology \& Computer Science at East Carolina University. He received his Associate of Applied Science in Military Studies from Community College of the Air Force; his BS in Professional Aeronautics from Embry Riddle Aeronautical University; and his Masters of Business Management: from Wayland Baptist University. He is currently teaching Instructor in the Information and Computer Technology Program; he is also Internship coordinator, responsible for student placement and supervision. His has developed and instructed courses and labs in Network Technologies and Network Operating Systems and is a Lead Cisco Networking Academy Instructor.

Address: College of Technology \& Computer Science, 205 Science and Technology Bldg, East Carolina University, Greenville, NC 27858; email: pickardj@ecu.edu; phone: 252-328-9646. 


\title{
Data in Depth: Web 3-D Technologies Provide New Approaches to the Presentation of Course Content
}

\begin{abstract}
For nearly two decades, the Web has provided the classroom with vast, ever-expanding volumes of browser-accessible information. As the web has evolved so too has our desire to become more involved with the process of content-creation and content-sharing. Now new web-based technologies look to provide smarter, more meaningful content and present that content with a new level of depth and interactivity. No longer are faculty and students browsing for information that is largely static; instead, these users are interacting through their threedimensional (3-D) proxies (their avatars) and are querying applications (semantic web agents) soliciting them to collect, filter, verify, correlate, and present answers to their queries. Yet, all of this capability is not without potential challenges.

There is an evolving need for faculty and students to find and build out new structure in their 3-D virtual surroundings that visually enables their content, making it more palatable to the user while presenting it in a 3-D format verses the typical 2-D format that has been the mainstay for the past two decades. With the maturation of virtual world (3-D Web) and semantic web technologies, the web-based content available in the classroom increases exponentially and takes

on a new look. Following a brief overview of these two technologies and their overall impact in the classroom, this article presents several practical approaches for presenting course content in 3-D Web environments based on recent implementation efforts. In-World lectures and lab assignments, project team briefing sessions, student mentoring activities, and open conference forums are just a few of the areas discussed. Further discussions also focus on setup and future evaluation studies planned in the near-term to further evaluate course content presentation techniques.
\end{abstract}

\section{Introduction}

The evolution of three-dimensional (3-D) Web technologies has evolved into a broad collection of virtual world online communities often taking the form of computer-based simulated environments allowing users to immerse, interact, share experiences, create and co-produce with other users. The concept of web-based 3-D virtual environments has become synonymous with the term virtual worlds where users take the form of avatars visible to others graphically in 3-D computer-generated spaces. As a 3-D web interface, virtual worlds provide users with some unique capabilities. On the surface, a web interface has four basic design elements in a 2-D space: line, color, shape and direction; add a third dimension to that equation gives us the additional elements of form and texture. ${ }^{1} \quad$ Although the early emphasis has been on building out virtual worlds and providing for a growing demand for more social context and interaction, there are some recent indicators that show a growing interest in the use of virtual world's as vehicles for presenting content. ${ }^{2}$

Over the past several years academics have begun to build and evaluate various virtual world environments with the goals of providing visually acceptable and meaningful meeting places that 
students and faculty can use to gather and communicate in. However, to date that communication has been mostly limited to providing an environment for social interaction with minimal emphasis on the presentation of content within these environments. With the continuing emergence of web based technologies like the Semantic Web with all of its volumes of "more meaningful" data and often auto-generated content, there is a growing need for academia to maximize the use of Web 3-D spaces like virtual worlds to present this content in more effective ways. Discussions include early use of Web 3-D spaces and identification of viable virtual environments to build and interact in, the growing interest in content presentation, unique challenges to presenting in 3-D and some thoughts on moving forward with these technologies.

\section{Early Use of Web 3-D Spaces}

In recent years, the use of 3-D Web environments has witnessed tremendous growth to a point where the World Wide Web (WWW) currently supports nearly 600 million virtual world users worldwide with nearly half of those falling into the up and coming 10-15 year old age group. ${ }^{3}$ Current reports also indicate that nearly 150 virtual world environments in existence today with that number expected to grow to 900 or more within the next three years. ${ }^{4}$ The past two decades the Web has witnessed a 1990's era, often referred to as Web 1.0, that focused mostly on readonly content and static HTML-based websites with early websites that were generally not interactive and Web technologies concentrating mostly on linking documents between the everincreasing numbers of Web servers.

Over the past ten years Web users have shifted gears with increased use of web service and other related technologies that have focused heavily on user-generated content. This recent period of Web usage, often been referred to as Web 2.0 or the Social Web, has proved users with more of a read-write Web capability allowing Web users to contribute or add to the Web content as well as consuming it through the use of various forums, blogs and social sites like Twitter, MySpace, YouTube, and Facebook. ${ }^{5}$ In a time when the Web enables all its users to be Web publishers, academics are witnessing an explosion of content that is being exchanged between faculty and students, up and down both formal and informal course channels of communications. These trends of social interaction, auto-content generation, are all lead-in's to what is now referred to as the Web 3.0 or the Semantic Web.

Today, there are literally dozens of 3-D Web virtual worlds available. A review of the numerous available 3-D virtual worlds finds that most are intended more for providing entertainment and social networking. Some, such as Neverwinter Nights, are fantasy worlds were the player is the center of an epic tale, a war, or a quest. ${ }^{6}$ Others are places to meet, socialize, and make new friends. An example is the 3-D virtual world There that claims everyone is friendly and that they are either an old friend, a new friend, or an about-to-be-friend. ${ }^{7}$

However, virtual worlds can be more than just social networking platforms and have a potential to enhance classroom interaction as a distance communication and collaboration media. The Immersive Education Initiative is an international collaboration of universities, colleges, research institutes, consortia and companies that are working together to define and develop open standards, best practices, platforms, and communities of support for virtual reality and gamebased learning and training systems. The Immersive Education website showcases three open 
source virtual worlds that engage students the same way video games grab and keep the attention of players: Project Wonderland, Second Life, and Cobalt. ${ }^{8}$

Sun's Project Wonderland is an open source 3D virtual world designed for business communication and collaboration. Wonderland allows "users to communicate and share live desktop applications and documents and conduct real business." Organizations can run their own Wonderland server or can download the Wonderland client and connect to publicly available Wonderland worlds. ${ }^{9}$

Second Life, also an open source platform, boasts the Internet's largest user-created 3-D virtual world community. Since opening to the public in 2003, it has grown explosively and today is inhabited by millions of Residents from around the globe. According to Virtual Worlds News users around the world have spent more than one billion hours in Second Life to date, and that there is now more than 270 terabytes of content contained in the virtual world. ${ }^{10}$ Second life is also host to the largest educational presence in any virtual world, the New Media Consortium (NMC) Campus Project. The project involves more than 150 colleges and universities and more than 12,000 educators. ${ }^{11}$

Cobalt is an open source virtual workspace browser and construction toolkit for accessing, creating, and publishing hyperlinked multiuser virtual environments. The vision behind Cobalt is to enable researchers, educators, students, and others to interact within interlinked, public or private $3 \mathrm{D}$ virtual workspaces that are customizable. ${ }^{12}$

In addition to the three open source virtual worlds discussed above, ActiveWorlds which is one of the oldest of the 3-D virtual worlds has responded to the demand from educators by launching the ActiveWorlds Educational University (AWEBU). The AWEDU is a unique educational community that makes the Active Worlds technology available to educational institutions, teachers, students, and individual programs. It is an entire Active Worlds Universe dedicated to exploring the educational applications of the ActiveWorlds Technology; however, unlike the ActiveWorlds main grid, the Educational Universe requires licensing fees. ${ }^{13}$

\section{A Growing Interest In Content Presentation}

As a 3-D Web interface, virtual world environments provide Web users with some unique capabilities. Virtual World environments provide six distinct functionalities. First, there is the concept of 'shared space' where the environment allows multiple users to participate at once. The second functionality involves the use of a 'graphical user interface' where the environment depicts space visually. The third functionality asserts the need for the virtual environment to provide for a 'sense of immediacy' where interaction takes place in real-time. The fourth provides for a high level of 'interactivity' within the environment, thus allowing users to alter, develop, build, or submit customized the content. The fifth functionality notes that there is a 'level of persistence' in that the virtual world's existence continues regardless of whether the users are logged in. And the final aspect of functionality involves the concept of 'socialization and community' where the virtual environment not only allows for but also encourages the formation of in-world social groups and interaction. ${ }^{14}$ 
From a purely functional perspective, early virtual world efforts within academia have taken advantage of Web 3-D technologies in many areas including social presence, persistence and the visual presentation of the virtual environment. It is only nature to presume that early emphasis would be centered on the visual presentation or the building out these environments for pedagogical deployment. Fundamental to maximizing the capabilities of computer mediated systems is "the ability to tailor human communication processes to the application and group undertaking its application". 15

With virtual environments, the focus upfront centers on the need to develop virtual classroom and meeting spaces that not only replace the actual real world academic experiences, but also maximize the inherent unique functionalities that the new virtual world provides. Yet once the spaces are in place there comes the need to communicate course content; there inlays the impetus behind a growing interest in the use of 3-D Web environments as vehicles for presenting content. The new challenge for academics is the communicating in a new third dimension.

\section{Data-In-Depth: A 3-D Building Approach}

Lesko \& Hollingsworth (2009) noted that "it is not just the challenge of making the data more meaningful, we must also embrace a new era of data presentation; an era where 3-D web-based Virtual World environments provide users with a new sense of 'Data-in-Depth'". ${ }^{16}$ This section describes considerations made in preparation for a series of virtual classroom designs; it also incorporates some of the early virtual classroom and laboratory spaces developed within a newly established virtual campus. Once these spaces are built, class sessions are being conducted and the students are surveyed. It should be noted at this point, that most of this section is based on research observation and will require further case analysis to validate site building trends and tendencies.

The development of Web 3-D virtual world spaces for academic use presents unique challenges for both academics and their students. To present course content in more effective ways first requires an understanding of where the content comes from. Early use of the virtual environment Second Life and recent work with Sun Systems Project Wonderland provided a means for early design and testing of basic communication and collaborative toolset within a virtual 3-D world experience.

From both the communication and collaborative perspectives early student interactions generated many new insights into other areas where virtual classrooms and laboratories presenting new options for continued site development and growth. ${ }^{16}$ From these early studies it became clear that new approaches to presenting content were in order. The following outlines a series of 3-D depictions describing the typical evolutionary growth of a virtual class space based on authors observations over the past several years of site development activity.

As indicated earlier, over the past several years academics have begun to build and evaluate various virtual world environments with the goals of providing visually acceptable and meaningful meeting places. ${ }^{17}$ Recent observation has shown this to be a typical early phase of site development. The next series of figures describe a typical sequence of steps taken to building collaborative virtual 3-D learning spaces. Figure 1 shows an early established course 
meeting space with a heavy focus on providing students with a realistic look of a typical meeting area. This particular area has been used by students in project teams to provide weekly status briefings and for team and student mentoring sessions.

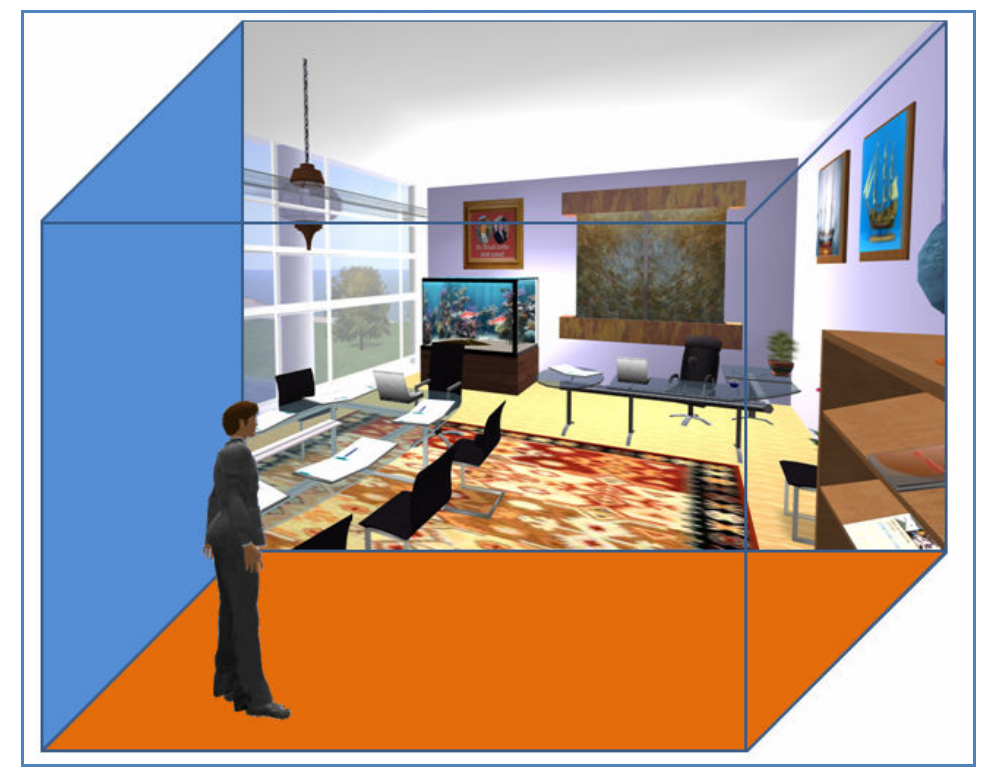

Figure 1: Visually Appealing Meeting Space

There can be a fine line between similarity to the real world (RW) and to the development of a virtual world (VW). The notion that academics might utilize the capabilities of current virtual technologies to mimic or even supplant RW classroom and instructional settings is not a new one. Hiltz \& Turoff (1993) noted early on that fundamental to maximizing the capabilities of computer mediated communication systems is "the ability to tailor human communication processes to the application and group undertaking its application". ${ }^{15}$ With virtual classroom environments, the focus upfront has centered on the need to develop virtual spaces that not only replicate actual RW academic experiences, but also maximizes the inherent functionalities that the new VW provides.

The next depiction Figure 2 describes the typical collaborative features utilized in virtual classroom sessions. Text chat boards, image depictions (including typical slide presentations), document viewers, and whiteboards are all common collaborative tools incorporated early in site developments. Of note here is the fact that all of these tools are merely replications of 2-D tools used in real world (RL) classrooms. Additionally, the content from these tools it typically housed within the virtual world solution itself and is not generally pulled from external resources such as other academic systems or the Internet. Content for slide presentations and document viewings are typically uploaded to the virtual world environment and activity such as text chats, and white-boarding is logged and maintained internally within the virtual world solution as well. 


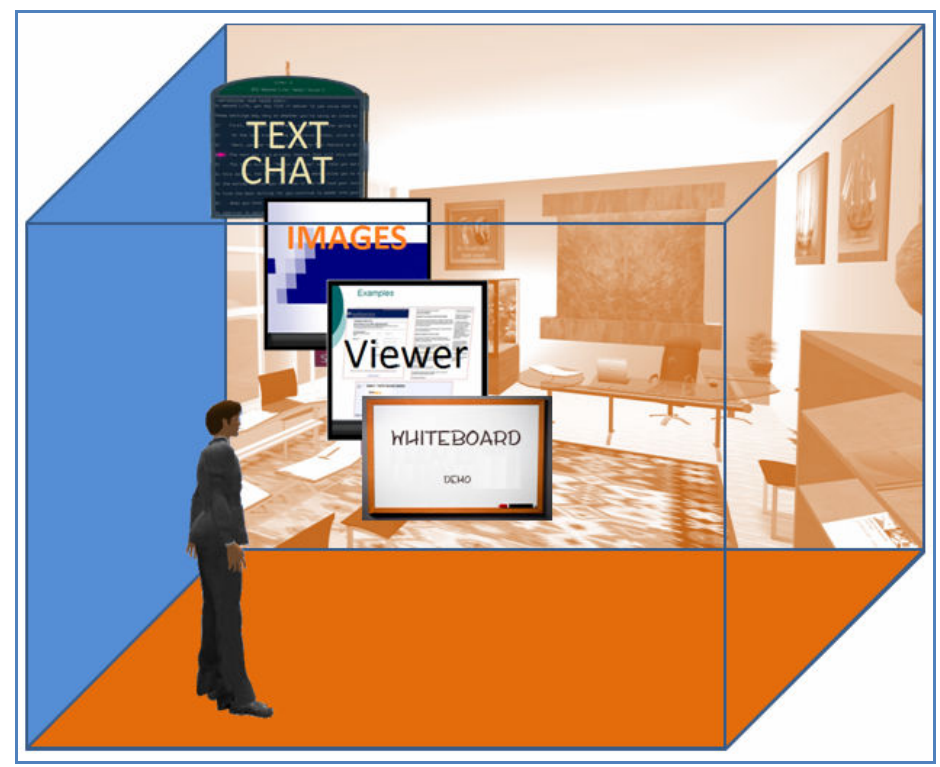

Figure 2: Basic In-World Collaboration

The next depiction Figure 3 describes the typical audio and video collaborative features incorporated beyond basic in-world collaboration functionalities. These may include use of voice chat, basic avatar body gesturing (i.e. pointing, raising hand, laughing, etc.) and use of video clips. As with basic in-world collaborations discussed previously, the content from background audio is typically housed within the virtual world solution itself and is not generally pulled from external resources. Content from voice chats and basic avatar action and gesturing is logged and maintained internally within the virtual world solution.

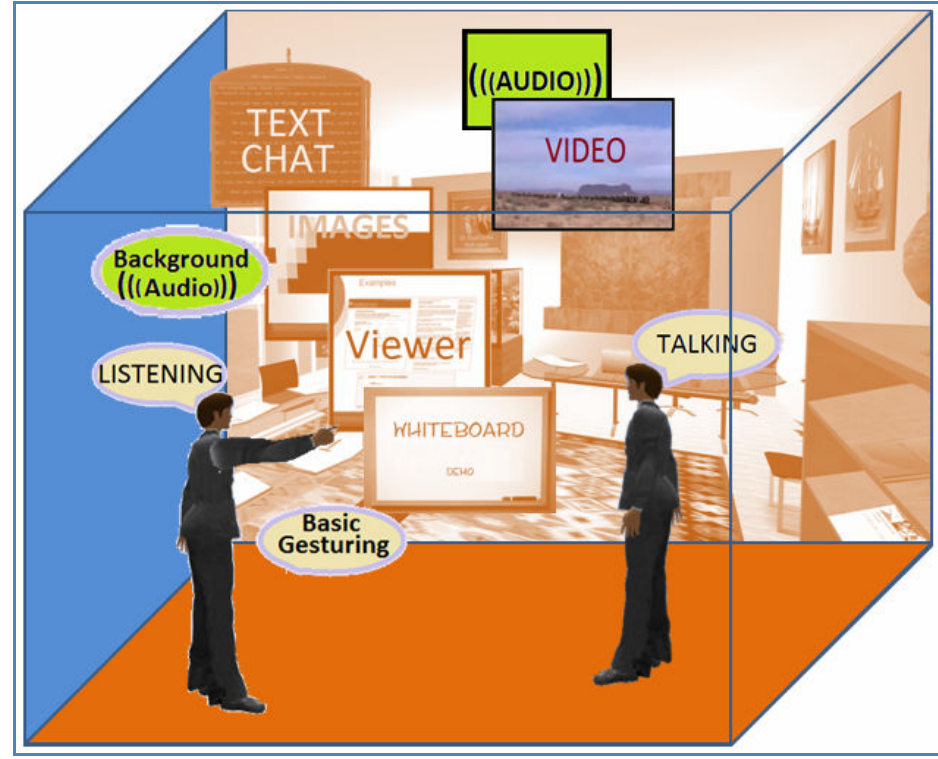

Figure 3: Audio, Video and Basic Gesturing Collaboration 
Where the content comes from is critical to any system implementation and operation. Up until this point the content has be typically housed with internally with the virtual 3-D solution. As with most of today's virtual worlds where content is housed in large scale infrastructures comprised of multiple servers, storage devices and networks is complex; however, most content is typically housed 'locally' within a corporately administered solution. Characteristics or this type of virtual world solution include single ownership of data and solution with common access control and storage retention policies. Internal content is typically created, stored and managed internally.

However, as discussed earlier Web users have shifted gears with increased use of web services and other related technologies focusing heavily on user-generated socio-centric content. This focus is on interacting with external content that are typically not housed within the virtual 3-D world solution. There is an increasing demand for rich data resources found across the web so access to Internet Browser capabilities in-world is becoming critical. Many of today academic sites, labs, classroom administration, library resources, etc. are all accessed via the internet. Along with basic browser functionality comes the ability to access and filter video and other social network feeds such as Twitter, YouTube, Facebook, etc. Additionally, teleconference capabilities provide another level of external collaboration for the classroom allowing for the incorporation of those who lack computer access but have telephone access to collaborate and interact with academic sessions including class lecture and labs, mentoring sessions, and group/team meetings.

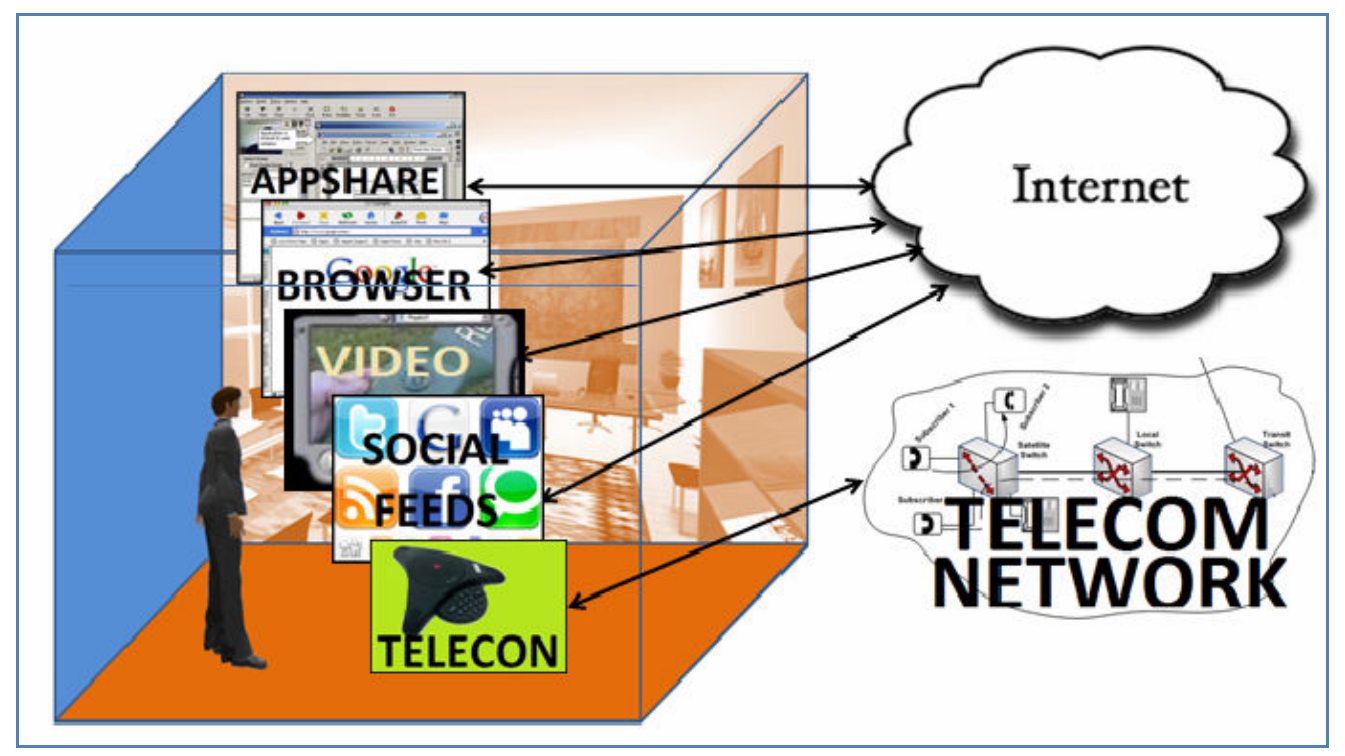

Figure 4: External Content Presentation an Collaboration

The final depiction, Figure 5, describes building 3-D virtual class space capable of automating the content collection process and generating unique content for academic delivery. An example of this might be similar to the way many online newspapers are being auto-generated today. Team projects or course assignments would generate rules for collection and assignment bots would perform the tasks of locating and presenting the content for students and faculty. From a 
more visual perspective, The ability to interact with data in-world and the present that data in 3$\mathrm{D}$ is also a 3-D classroom building consideration. Up to this point, most of the content has been presented via various 2-D common formats found in our daily interacts such as browsers, appshares, viewers, videos, etc. To maximize the use of 3-D space requires moving into the realm of 3-D content presentation and interaction.

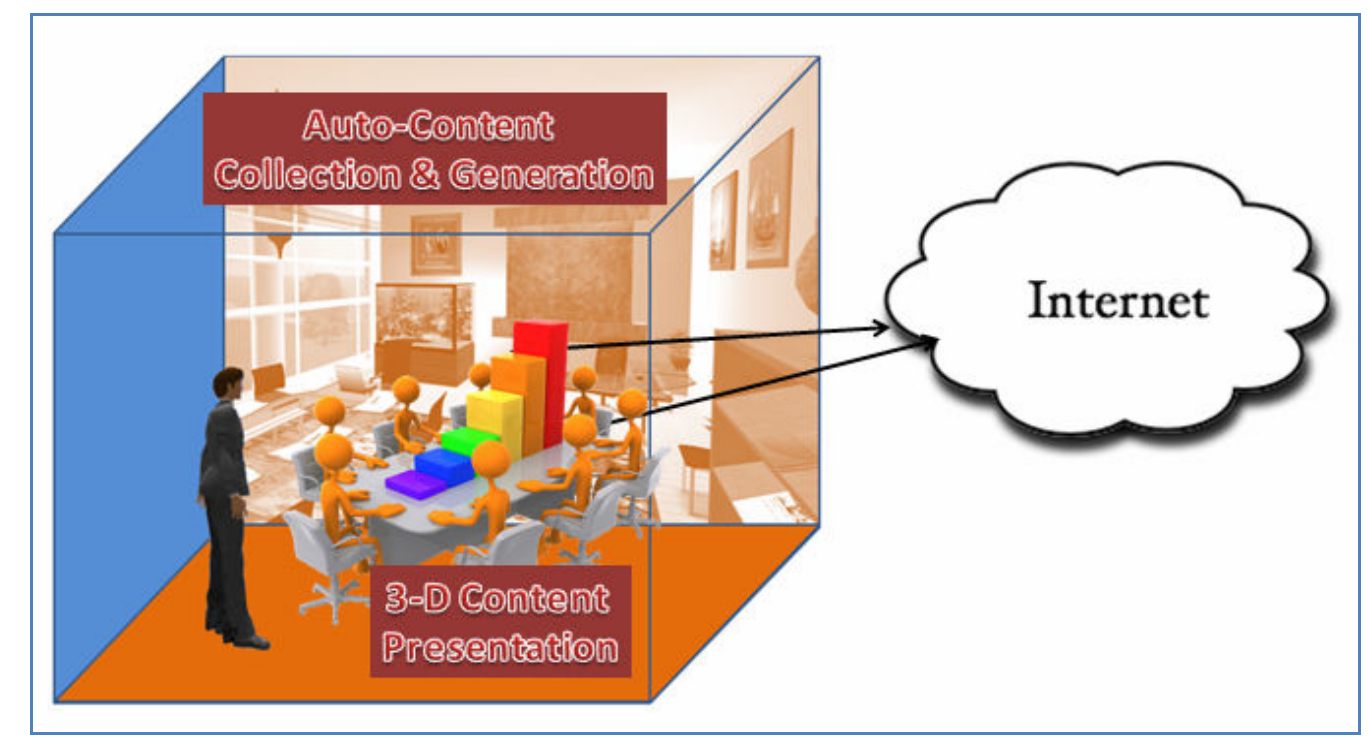

Figure 5. Auto-Content Collection and Generation with 3-D Content Presentation

\section{A Focus for Moving Forward}

Virtual 3-D Web environments provide faculty and students with communicative tools having endless possibilities. Virtual classroom workspaces should consider the aspects of course content exchange. Academics are there to provide the framework for the course that allows for the exchange of various types of course content to enable successful completion of each course objective. Creating virtual classrooms and meeting spaces that give students the freedom to explore and build their own learning environments is truly leading us into new uncharted territories. $^{16}$

Further research will focus on building 3-D virtual class space capable of automating the content collection process and generating unique content for academic delivery. Of further interest is the ability to collect objects (3-D in most cases) and case studies as virtual realities from multiple perspectives suggests a new methodology for research. Building environments that provide for these collections should prove beneficial to furthering the educational process and maximizing the virtual environmental spaces academic utility. Students working either individually, in pairs, in mentoring situation, or in teams to design and build objects that should enhance the virtual experience. Items built should have learning and educational value for other students. 


\section{Bibliography}

[1] J. Paul Getty Trust. The Elements of Art. The Getty. [Online] The J. Paul Getty Trust, 2009. [Cited: December 17, 2009.] http://www.getty.edu/education/teachers/building_lessons/elements.html.

[2] Burden, David. Toward Semantic Virtual Worlds - A Thinkpiece. www.converj.com. [Online] May 13, 2009. [Cited: September 3, 2009.] http://www.converj.com/sites/converjed/2009/05/toward_semantic_virtual_worlds.html.

[3] K Zero. K Zero: 260M Registered Accounts for the 10-15-Year-Old Virtual World Demo. VirtualWorldNews.com. [Online] February 09, 2009. [Cited: September 16, 2009.] http://www.virtualworldsnews.com/2009/02/k-zero-260m-registered-accounts-for-the-1015yearold-virtualworld-demo.html.

[4] Mitham, Nic. Growth forecasts for the Virtual Worlds sector. www.kzero.co.uk. [Online] September 12, 2009. [Cited: September 16, 2009.] http://www.kzero.co.uk/blog/?m=200909.

[5] Strickland, Jonathan. Is there a Web 1.0? computer.howstuffworks.com. [Online] 2009. [Cited: Sept 6, 2009.] http://computer.howstuffworks.com/web-10.htm.

[6] Bioware. Neverwinter Nights: What is Neverwinter nights? nwn.bioware.com. [Online] November 2009. [Cited: November 24, 2009.] http://nwn.bioware.com/about/description.html.

[7] There. There - What is There? Prod.there.com. [Online] November 2009. [Cited: November 26, 2009.] http://www.prod.there.com/info/whatisthere.

[8] Immersive Education. The Immersive Education Initiative. Immersiveeducation.org. [Online] November 2009. [Cited: November 24, 2009.] http://immersiveeducation.org/\#about_initiative.

[9] Sun Systems. lg3d-wonderland: Project Wonderland. lg3d-wonderland.dev.java.net. [Online] November 2009. [Cited: November 26, 2009.] http://lg3d-wonderland.dev.java.net/.

[10] Virtual World News. Linden Lab Releases Second Life Stats. Virtualworldnews.com. [Online] November 2009. [Cited: November 26, 2009.] http://www.virtualworldsnews.com/2009/09/linden-lab-release-secondlife-stats-onslaught.html.

[11] New Media Consortium. NMC Projects . nmc.org. [Online] November 2009. [Cited: November 26, 2009.] http://www.nmc.org/proj.

[12] Duke. What is Cobalt? - Duke Wiki. Wiki.duke.edu. [Online] November 2009. [Cited: November 26, 2009.] https://wiki.duke.edu/pages/viewpage.action?pageId=10717764.

[13] ActiveWorlds. Active Worlds Educational Universe Pricing Information. Activeworlds.com. [Online] November 2009. [Cited: November 24, 2009.] http://www.activeworlds.com/edu/awedu_pricing.asp.

[14] What is a Virtual World? Virtual Worlds Review. [Online] 2009. [Cited: Sept 10, 2009.] http://www.virtualworldsreview.com/info/whatis.shtml.

[15] Hiltz, S. R. and Turoff, M. The Network Nation: Human Communication via Computer. Reading, MA : MIT Press, 1993.

[16] Lesko, Charles and Hollingsworth, Yolanda. Compounding the Results: The Integration of Virtual Worlds with the Semantic Web. Journal of Virtual World Research. s.l. : [Article submitted for review], 2009.

[17] Lesko, Charles and Pickard, John. Design Considerations for Virtual Classroom and Laboratory Environments. San Antonio, TX : American Society for Engineering Education, 2009. 